\title{
EFECTO DEL ACETATO DE PLOMO SOBRE LA CAPACITACIóN ESPERMÁTICA hUMANA
}

\author{
Roy Anderson ${ }^{l}$ \\ Adriana Castillo $^{1}$ \\ Mauricio Gonzales ${ }^{1}$ \\ Flor de María Madrid ${ }^{2}$
}

\begin{abstract}
RESUMEN
El plomo es un agente contaminante presente en el ambiente producto de la minería, manufactura industrial y la quema de combustibles fósiles. Se ha observado que individuos expuestos ocupacionalmente a plomo presentan disfunción de las glándulas sexuales, disminución de la concentración espermática, disminución de la motilidad y algunas otras alteraciones.

El objetivo de este estudio fue evaluar el efecto del acetato de plomo sobre la capacitación espermática. El medio que se utilizó para la capacitación fue Tyrode libre de $\mathrm{Ca}^{+}$, suplementado con Albumina de Bovino Sérica (BSA) por 30 minutos a $37^{\circ} \mathrm{C}, 6 \% \mathrm{CO}$ y bajo concentraciones de $\mathrm{Pb}\left(\mathrm{CH}_{3} \mathrm{COO}\right)_{\text {a }} 0.6 \mu \mathrm{g} / \mathrm{dL} ; 6 \mu \mathrm{g} / \mathrm{dL}$ y $66 \mu \mathrm{g} / \mathrm{dL}$. Se realizó la valoración de la motilidad y vitalidad espermática pre y post capacitación. Como resultados se obtuvo una disminución en la vitalidad con medias de 17.99 y 46.99 para la concentración de $66 \mu \mathrm{g} / \mathrm{dL}$, además se observaron efectos en la motilidad, con medias de 1.2 y 26 a la misma concentración En conclusión, el acetato de plomo $\mathrm{Pb}\left(\mathrm{CH}_{3} \mathrm{COO}\right)_{2}$ tiene efectos, sobre la motilidad y vitalidad, disminuyendo los porcentajes óptimos durante la capacitación espermática.
\end{abstract}

Palabras clave: Acetato de plomo, vitalidad, motilidad lineal.

\begin{abstract}
Lead is a contaminant in the environment from mining, industrial manufacturing and burning fossil fuels. It has been observed in individuals occupationally exposed to lead have sex gland dysfunction, decreased sperm count, decreased sperm motility and some alterations.

The aim of this study was to evaluate the effect of lead acetate on sperm capacitation. The medium used for the training was free Tyrode $\mathrm{Ca}+$ supplemented with Serica bovine albumin (BSA) for 30 minutes at $37^{\circ} \mathrm{C}$, $6 \% \mathrm{CO} 2$, low concentrations $0.6 \mu \mathrm{g} / \mathrm{dL} ; 66 \mu \mathrm{g} / \mathrm{dL} 6 \mu \mathrm{g} / \mathrm{dL}$ and $\mathrm{Pb}(\mathrm{CH} 3 \mathrm{COO}) 2$. We conducted the assessment of sperm motility and vitality pre and post training. Results were obtained as a decrease in the average vitality 17.99 and 46.99 for $66 \mu \mathrm{g} / \mathrm{dL}$ concentration, besides effects were observed in motility, with averages of 1.2 and 26 at the same concentration. In conclusion, lead acetate $\mathrm{Pb}(\mathrm{CH} 3 \mathrm{COO}) 2$ has effects on reducing the percentage motility and vitality for optimal sperm capacitation.
\end{abstract}

Keywords: lead acetate, vitality, linear motility.

\footnotetext{
${ }^{1}$ Laboratorio de Biotecnología y Fisiología Animal, Facultad de Ciencias Biológicas, Universidad Ricardo Palma.
}

${ }^{2}$ Instituto de Recursos Naturales y Ecología, Universidad Ricardo Palma. 


\section{INTRODUCCIóN}

La contaminación es un problema real y muy preocupante en nuestros tiempos. Entre los contaminantes más comunes y peligrosos se encuentra el Plomo $(\mathrm{Pb})$, un metal tóxico que también encontramos en forma natural, y que por ser un elemento básico, no se puede disociar o degradar. Existe un amplio espectro de productos derivados del plomo, estos dejan a la población expuesta a sus efectos nocivos para la salud, quedando como única alternativa, la minimización de su empleo y el control de las emisiones de éste al medio ambiente

En el presente trabajo utilizaremos no el elemento plomo exclusivamente, pues su toxicidad es tal que al trabajar con semen directamente correríamos el riesgo de matar a los espermatozoides con el solo contacto de estos con el metal, sino con una solución de Acetato de Plomo, la cual se encuentra en una variedad de productos de uso cotidiano, de forma aparentemente "inofensiva", como lo son las pinturas, textiles, murales, tintes para el cabello en la industria de la belleza, así como en juguetes para los niños.

Por lo expuesto evaluaremos si al someter los espermatozoides a diferentes concentraciones de acetato de plomo, antes y después de su capacitación in vitro, podremos verificar el efecto del plomo sobre la capacitación espermática y su probable repercusión en la salud reproductiva.

\section{MATERIAL Y MÉTODOS}

\section{Recolección del eyaculado}

Para llevar a cabo el análisis se recolectaron 3 muestras de eyaculado humano de donantes diferentes de la Facultad de Ciencias Biológicas, con un periodo de abstinencia sexual de 4 días, además se tomaron datos como la edad, realización de actividades físicas (deporte, artísticas, otros) para determinar el estrés de la persona en cada uno de los donantes y los valores del espermograma de los cuales solo consideramos los índices microscópicos entre los cuales se encuentra la concentración espermática, la motilidad y la vitalidad.

Los valores a considerar como criterios de normalidad presentados por la OMS son: volumen 1.5 a más mL, pH 7.2 - 7.8 concentración espermática $\geq 15$ millones, motilidad $\geq 40 \%$, vitalidad $\geq 58 \%$, morfología $\geq 4 \%$ normales, de los cuales solo consideraremos vitalidad y motilidad para esta investigación.

\section{Preparación del Medio de Capacitación}

Utilizamos una Solución Tyrode libre de Ca, luego, adaptamos las cantidades de los reactivos que obtuvimos desde $1000 \mathrm{~mL}$ a $250 \mathrm{~mL}$. Luego, agregamos BSA a $125 \mathrm{~mL}$ de nuestra Solución Tyrode libre de $\mathrm{Ca}$, teniendo en cuenta el agregar $20 \mu \mathrm{g} / \mathrm{dL}$.

\section{Tratamiento del eyaculado.}

\section{A. Medición del pico de la hiperactividad}

Se tomó un eyaculado de cada individuo y se diluyó en el medio hasta lograr una concentración de 200-500 espermatozoides por mililitro; Luego capacitamos estos espermatozoides con BSA y realizamos el conteo de espermatozoides hiperactivos a diferentes tiempos, hasta determinar en cual encontramos un pico de espermatozoides hiperactivos capacitados, el resultado se diò a los 30 minutos para los 3 donantes.

\section{B. Determinación de las concentraciones}

\section{del $\mathrm{Pb}\left(\mathrm{Ch}_{3} \mathrm{COO}\right)_{2}$}

Trabajamos con 3 diferentes concentraciones, siguiendo lo indicado por Mendiola et al, pues encontraron como máximo $66.5 \mu \mathrm{g} / \mathrm{dL}$ de plomo en el semen, tomando este dato como nuestra primera concentración, para luego realizar diluciones siendo las concentraciones finales: $0.6 \mu \mathrm{g} / \mathrm{dL} ; 6 \mu \mathrm{g} / \mathrm{dL}$ y $66 \mu \mathrm{g} / \mathrm{dL}$.

C. Evaluación de los efectos del $\mathrm{Pb}\left(\mathrm{Ch}_{3} \mathrm{COO}\right)_{2}$, en muestras capacitadas (simulan exposición en la mujer) y no capacitadas (simulan exposición en el hombre).

Dividimos las muestras previamente diluidas en dos grupos para el tratamiento con $\mathrm{Pb}\left(\mathrm{CH}_{3} \mathrm{COO}\right)_{2}$ :

\section{a. Muestras no capacitadas}

Del primer grupo se extrajo el eyaculado del individuo 1, se tomaron 3 alícuotas. A cada alícuota se le agregó $\mathrm{Pb}\left(\mathrm{CH}_{3} \mathrm{COO}\right)_{2}$, para lograr las concentraciones anteriormente determinadas, y se dejó reaccionar durante 10 minutos. Así procedimos con las muestras de los 2 individuos restantes. Luego agre- 
gamos a todas las muestras BSA para capacitar a los espermatozoides y dejamos incubar durante el tiempo determinado como pico de motilidad lineal rápida que calculamos para cada individuo (30 min). Posteriormente realizamos el conteo de cada muestra siguiendo los criterios de vitalidad y motilidad lineal rápida.

\section{b. Muestras capacitadas.}

Del segundo grupose extrajoel eyaculadodel individuo 1, se tomaron 3 alícuotas. A cada alícuota se le agregò BSA para capacitar a los espermatozoides y luego le agregamos $\mathrm{Pb}\left(\mathrm{CH}_{3} \mathrm{COO}\right)_{2}$, para lograr las concentraciones anteriormente determinadas. Del mismo modo procedimos con las muestras de los 2 individuos restantes y se dejó incubar durante el tiempo determinado como pico de motilidad lineal rápida que calculamos para cada individuo (30min). Posteriormente realizamos el conteo de cada muestra siguiendo los criterios de vitalidad y motilidad lineal rápida.

\section{Procesamiento de datos.}

Los datos fueron recopilados y procesados, primero, en función a sus parámetros descriptivos, y luego por medio del programa SPSS 20.0, a los cuales aplicamos el método ANOVA univariable comparando: para la Vitalidad, las relaciones entre donantes VS las concentraciones de $\mathrm{Pb}\left(\mathrm{CH}_{3} \mathrm{COO}\right)_{2}$; y para la Motilidad VS el comportamiento de las muestras.

\section{RESULTADOS}

La media de espermatozoides incubados únicamente con la solución Tyrode libre de $\mathrm{Ca}$, sin capacitar con BSA, es $\mathrm{X} 1=61.6$, en contraste con su similar capacitado que nos da $\mathrm{X} 2=81.5$, pudiendo interpretar estos datos diciendo que el BSA aumenta la capacitación in vitro en nuestra muestra en aproximadamente un $20 \%$.

Luego al agregar $\mathrm{Pb}\left(\mathrm{CH}_{3} \mathrm{COO}\right)_{2}$ en nuestra solución en las distintas concentraciones ya calculadas $(66 \mu \mathrm{g} / \mathrm{dL} ; 6 \mu \mathrm{L} / \mathrm{dL}$ y $0.6 \mu \mathrm{L} / \mathrm{dL})$ respectivamente, obtuvimos como medias $\mathrm{X} 1 C 1=17.99$; $\mathrm{X} 1 C 2=$ $28.99 \times 1 C 3=47.33$ para las muestras no capacitadas con BSA y luego obtuvimos las medias: $\mathrm{X} 2 \mathrm{C1}=$ 46.09; $\mathrm{X} 2 C 2=60.77 ; \mathrm{X} 2 C 3=70.86$ para las muestras capacitadas con BSA. Así, podemos entender en estos datos, como en el primer grupo las medias de vitalidad son considerablemente inferiores en contraste con el segundo grupo.

De igual manera trabajamos con la motilidad lineal rápida; en este caso, obtuvimos las medias para las muestras control pre y post capacitadas respectivamente: $\mathrm{X} 1=13.33$; $\mathrm{X} 2=62.1$; observando que la diferencia de motilidad lineal rápida si es muy notoria en ambos grupos, pues està directamente afectada por el BSA, siendo este tipo de motilidad la que permite la fertilización.

Observamos las medias para la motilidad lineal rápida a diferentes concentraciones de $\mathrm{Pb}\left(\mathrm{CH}_{3} \mathrm{COO}\right)_{2}: \mathrm{X} 1 C 1=1.2 ; \mathrm{X} 1 C 2=3.43$; $\mathrm{X} 1 C 3=$ 5.77 para las muestras no capacitadas con BSA; y $\mathrm{X} 2 C 1=26 ; \mathrm{X} 2 C 2=44.77 ; \mathrm{X} 2 C 3=49.66$ para las muestras capacitadas con BSA.

Estos datos nos indican el gran efecto que tiene el $\mathrm{Pb}\left(\mathrm{CH}_{3} \mathrm{COO}\right)_{2}$ sobre la motilidad de los espermatozoides, y su influencia en la fertilidad masculina.

En la Tabla 1 se puede observar la vitalidad y motilidad lineal rápida de los espermatozoides capacitados y no capacitados. Se aprecia la tendencia hacia la disminución de la motilidad lineal rápida y vitalidad respecto al aumento de la concentración del $\mathrm{Pb}\left(\mathrm{CH}_{3} \mathrm{COO}\right)_{2}$.

\begin{tabular}{|c|c|c|}
\hline$\left[\mathrm{Pb}\left(\mathrm{CH}_{3} \mathrm{COO}\right)_{2}\right]$ & Vitalidad & $\begin{array}{l}\text { Motilidad } \\
\text { Lineal rápida }\end{array}$ \\
\hline \multicolumn{3}{|c|}{ Sin capacitar } \\
\hline Control & 61.6 & 13.33 \\
\hline $0.66 \mu \mathrm{g} / \mathrm{dL}$ & 47.33 & 5.77 \\
\hline $6.6 \mu \mathrm{g} / \mathrm{dL}$ & 28.99 & 3.43 \\
\hline $66 \mu \mathrm{g} / \mathrm{dL}$ & 17.99 & 1.2 \\
\hline \multicolumn{3}{|c|}{ capacitados } \\
\hline Control & 81.5 & 62.1 \\
\hline $0.66 \mu \mathrm{g} / \mathrm{dL}$ & 70.86 & 49.66 \\
\hline $6.6 \mu \mathrm{g} / \mathrm{dL}$ & 60.77 & 44.77 \\
\hline $66 \mu \mathrm{g} / \mathrm{dL}$ & 46.09 & 26 \\
\hline
\end{tabular}


En la Tabla 2 se puede observar la variación de la vitalidad en función a la concentración de $\mathrm{Pb}\left(\mathrm{CH}_{3} \mathrm{COO}\right)_{2}$ en tres distintos donantes. Al aumentar la concentración de $\mathrm{Pb}\left(\mathrm{CH}_{3} \mathrm{COO}\right)_{2}$ disminuye la vitalidad de los espermatozoides.

\begin{tabular}{|c|c|c|c|c|c|c|c|c|c|c|c|c|}
\hline & \multicolumn{12}{|c|}{ Vitalidad } \\
\hline & \multicolumn{3}{|c|}{ Control } & \multicolumn{3}{|c|}{$66 \mu \mathrm{g} / \mathrm{dL}$} & \multicolumn{3}{|c|}{$6 \mu \mathrm{g} / \mathrm{dL}$} & \multicolumn{3}{|c|}{$0.6 \mu \mathrm{g} / \mathrm{dL}$} \\
\hline & \multicolumn{12}{|c|}{ No Capacitados } \\
\hline Donante & Media & Varianza & DS & Media & Varianza & DS & Media & Varianza & DS & Media & Varianza & DS \\
\hline 1 & 70 & 14750 & 121.44 & 23.66 & 1745 & 41.77 & 35 & 3753 & 61.26 & 44.66 & 6058 & 77.83 \\
\hline 2 & 62 & 11630 & 107.84 & 15.33 & 738 & 27.16 & 27.66 & 2337 & 48.34 & 43 & 5661 & 75.23 \\
\hline \multirow[t]{2}{*}{3} & 53 & 9225 & 96.04 & 5 & 725 & 26.92 & 24.33 & 1801 & 42.43 & 54.33 & 9041 & 95.08 \\
\hline & \multicolumn{12}{|c|}{ Capacitados } \\
\hline 1 & 81 & 19811 & 140.75 & 21.3 & 1386 & 37.22 & 51.33 & 8246 & 90.8 & 66.66 & 13352 & 115.55 \\
\hline 2 & 91 & 24857 & 157.66 & 53.66 & 8753 & 93.55 & 58 & 10130 & 100.64 & 69.33 & 144334 & 379.91 \\
\hline 3 & 72.66 & 15954 & 126.3 & 63.33 & 12050 & 109.77 & 73 & 16005 & 126.51 & 76.6 & 17652 & 132.86 \\
\hline
\end{tabular}

En la Tabla 3 se puede observar la variación de la motilidad lineal rápida en función a la concentración de acetato de plomo en tres distintos donantes. Al aumentar la concentración de $\mathrm{Pb}\left(\mathrm{CH}_{3} \mathrm{COO}\right)_{2}$ disminuye la motilidad lineal rápida.

\begin{tabular}{|c|c|c|c|c|c|c|c|c|c|c|c|c|}
\hline & \multicolumn{12}{|c|}{$\begin{array}{l}\text { Tabla 3. Parámetros descriptivos referidos a la Motilidad lineal rápida de los espermatozoides sometidos a los efectos de } \\
\mathrm{Pb}\left(\mathrm{CH}_{3} \mathrm{COO}\right)_{2}\end{array}$} \\
\hline & \multicolumn{12}{|c|}{ Motilidad } \\
\hline & \multicolumn{3}{|c|}{ Control } & \multicolumn{3}{|c|}{$66 \mu \mathrm{g} / \mathrm{dL}$} & \multicolumn{3}{|c|}{$6 \mu \mathrm{g} / \mathrm{dL}$} & \multicolumn{3}{|c|}{$0.6 \mu \mathrm{g} / \mathrm{dL}$} \\
\hline & \multicolumn{12}{|c|}{ No Capacitados } \\
\hline Donante & Media & Varianza & DS & Media & Varianza & DS & Media & Varianza & DS & Media & Varianza & DS \\
\hline 1 & 12 & 446 & 21.11 & 2.3 & 27 & 5.19 & 7 & 153 & 12.36 & 9 & 245 & 15.65 \\
\hline 2 & 9.33 & 270 & 16.43 & 0.66 & 2 & 1.41 & 2.3 & 21 & 4.58 & 6.33 & 141 & 11.87 \\
\hline \multirow[t]{2}{*}{3} & 18.66 & 1048 & 32.37 & 0.66 & 2 & 1.41 & 1 & 3 & 1.73 & 2 & 14 & 3.74 \\
\hline & \multicolumn{12}{|c|}{ Capacitados } \\
\hline 1 & 80.33 & 19445 & 139.44 & 23 & 1781 & 42.2 & 35.66 & 4509 & 67.14 & 42 & 6054 & 77.8 \\
\hline 2 & 58.66 & 10546 & 102.69 & 34 & 3494 & 59.11 & 37.66 & 4269 & 65.33 & 51 & 7827 & 88.47 \\
\hline 3 & 47.33 & 6766 & 82.25 & 21 & 1337 & 36.56 & 61 & 11261 & 106.11 & 56 & 9470 & 97.31 \\
\hline
\end{tabular}

Utilizando el método estadístico ANOVA univariable, obtuvimos las siguientes gráficas:

\section{Para las muestras no capacitadas:}

En el gráfico 1, observamos el comportamiento de los donantes en función de las concentraciones, y vemos como el porcentaje de espermatozoides vivos disminuye a medida que va aumentando la concentración de $\mathrm{Pb}\left(\mathrm{CH}_{3} \mathrm{COO}\right)_{2}$. 
Gráfico 1. Medias marginales estimadas de \% de espermatozoides vivos.

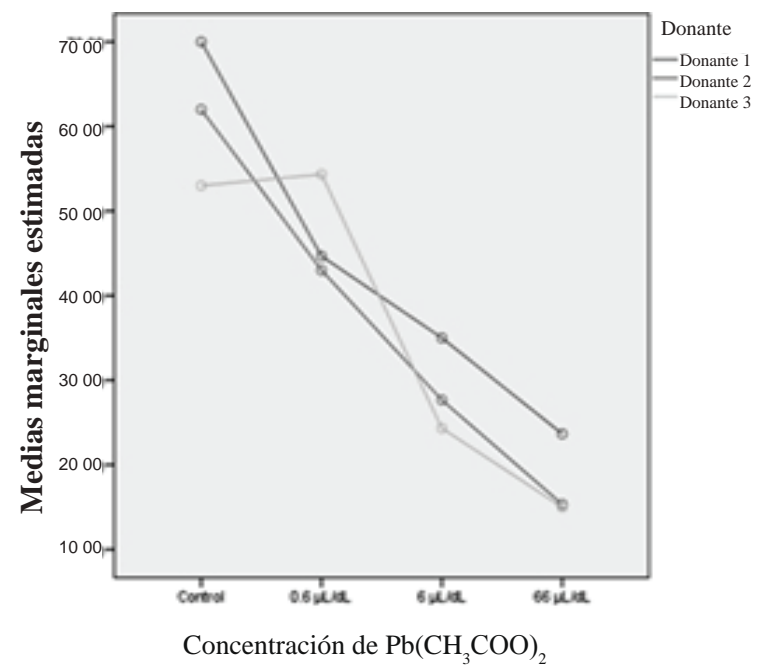

En el gráfico 2, observamos como nuestra concentración 1 afecta dramáticamente la motilidad lineal rápida, y como se va aumentando esta, a medida que se disminuye la concentración de $\mathrm{Pb}\left(\mathrm{CH}_{3} \mathrm{COO}\right)_{2}$.

Gráfico 2. Medias marginales estimadas de \% de espermatozoides con motilidad lineal rápida.

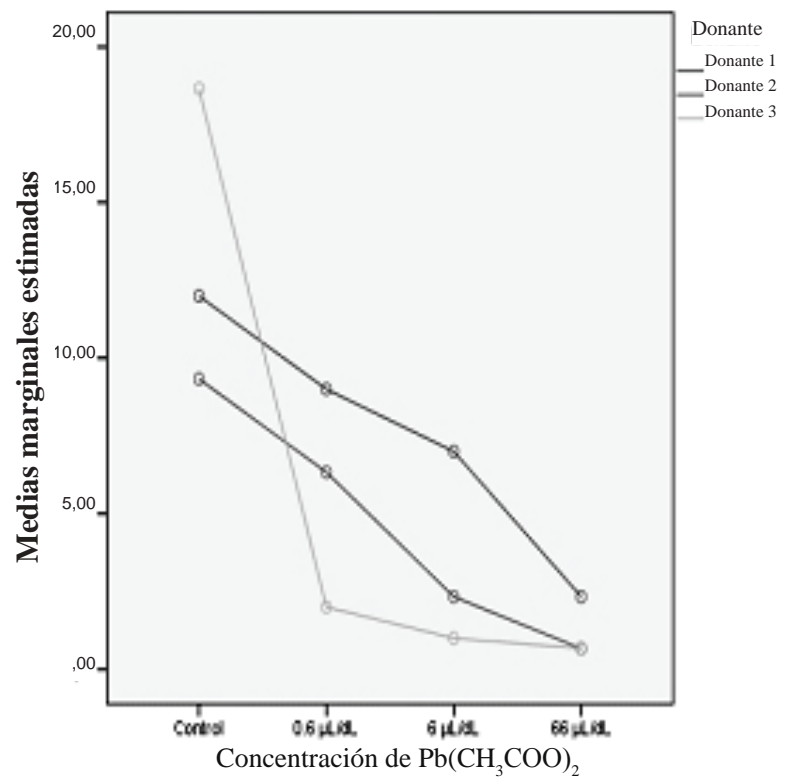

\section{Para las muestras capacitadas:}

En el gráfico 3, observamos cómo no existe relación alguna entre donantes, pero vemos como se comportan de manera similar frente al $\mathrm{Pb}\left(\mathrm{CH}_{3} \mathrm{COO}\right)_{2}$, en las concentraciones con las que se trabajò, observando la misma tendencia a la caída dramática de la vitalidad con las concentraciones permitidas.
Gráfico 3. Medias marginales estimadas de \% de espermatozoides vivos.

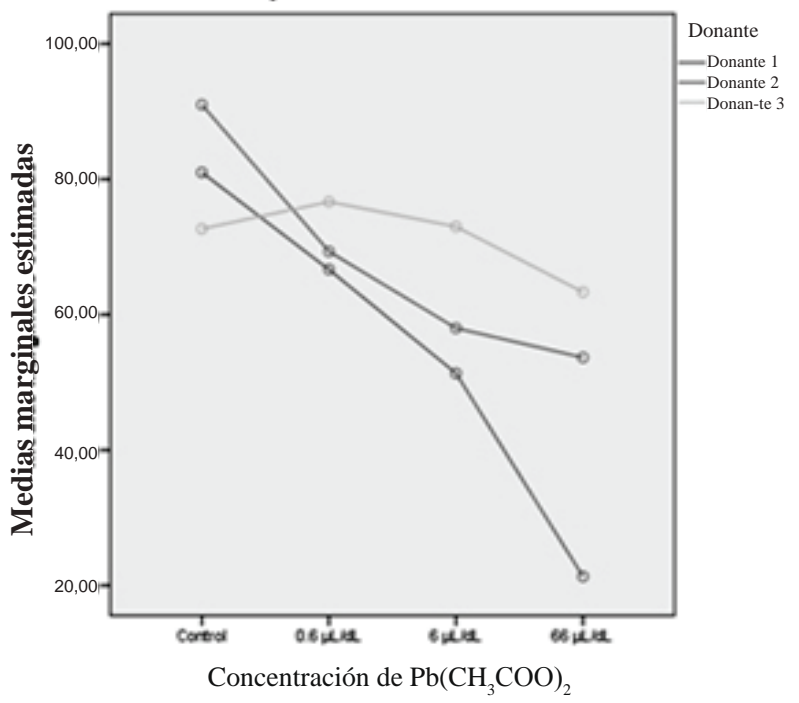

En el gráfico 4, observamos cómo se comportan los donantes (eyaculados) en relación a las concentraciones decrecientes de $\mathrm{Pb}\left(\mathrm{CH}_{3} \mathrm{COO}\right)_{2}$ con las que trabajamos, y como estas afectan de manera más puntual la motilidad lineal rápida.

Gráfico 4. Medias marginales estimadas de \% de espermatozoides con motilidad lineal rápida.

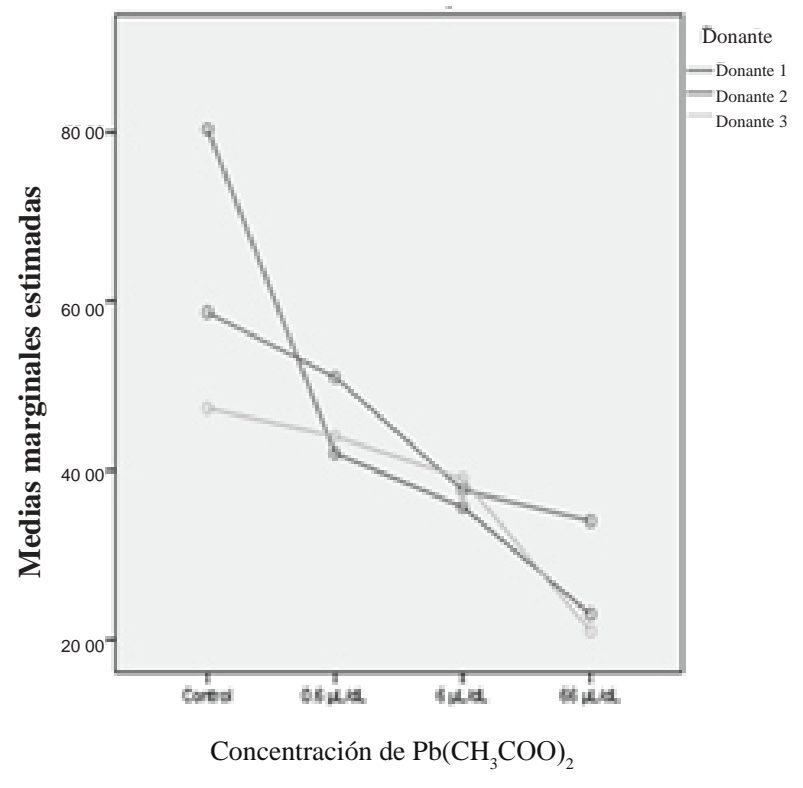

\section{DISCUSIóN}

Los valores normales de la OMS establecen 58\% de espermatozoides vivos en las muestras de eyaculado humano, en comparaciòn con nuestros resultados, en los que obtuvimos como vitalidad media $61,6 \%$ en los espermatozoides del grupo control no capa- 
citados, esto puede deberse a la dieta, a la actividad física que hayan realizado $\mathrm{u}$ otros factores externos.

Los valores normales de la OMS establecen un rango de 3 a $20 \%$ de espermatozoides con motilidad lineal rápida en las muestras de eyaculado humano, coincidiendo con nuestros resultados en los que obtuvimos como media $13,33 \%$ en los espermatozoides del grupo control no capacitados, lo cual indica que el valor se encuentra dentro de un rango establecido a nivel mundial.

Los resultados que muestran disminución de la vitalidad y motilidad lineal rápida se sustentan en investigaciones como las de Huidobro que en el 2010, expuso las causas de la infertilidad masculina, desde una perspectiva fisiológica abarcando los aspectos toxicológicos entre los cuales encontramos al $\mathrm{Pb}$ como uno de los principales metales pesados causantes de este mal. Cabe señalar además, que en el 2007 , Mendiola encontró $66.5 \mu \mathrm{g} / \mathrm{dL}$ de plomo en semen como valor máximo en profesionales expuestos. Asì como también se corrobora con los resultados obtenidos por Méndez en el 2012.

Considerando que la capacitación espermática in vivo, se genera en el oviducto femenino y si se reúnen las condiciones que hemos simulado in vitro en el laboratorio, podemos inferir que los resultados obtenidos en esta investigación puedan coincidir con la contaminación por acetato de plomo $\mathrm{Pb}(\mathrm{CH} 3 \mathrm{COO}) 2$, en individuos expuestos a concentraciones similares en donde el eyaculado que este produzca, disminuirá considerablemente la vitalidad y motilidad de sus espermatozoides. Coincidiendo con los estudios llevados a cabo por Yucra en el 2008, revelando que la exposición crónica al plomo en el varón incluye reducción de la libido, alteración en la espermatogénesis, dando como resultado una disminución en las tasas de fertilidad.

\section{CONCLUSIONES}

- El incremento de las concentraciones de $\mathrm{Pb}(\mathrm{CH} 3 \mathrm{COO}) 2$, disminuye la vitalidad y la motilidad lineal rápida en espermatozoides capacitados y no capacitados.

- Los efectos del $\mathrm{Pb}(\mathrm{CH} 3 \mathrm{COO}) 2$ son similares en los distintos eyaculados evaluados.

- El Pb(CH3COO)2, es tóxico para los espermatozoides humanos, afectando la capacitación espermática.

- Los espermatozoides son buenos bioindicadores frente a la contaminación por $\mathrm{Pb}(\mathrm{CH} 3 \mathrm{COO}) 2$.

\section{REFERENCIA BIBLIOGRÁFICA}

ASTETE J., Caceres W., Gastañaga M., Lucero M., Sabastizaga I., Oblitas T., Pari J., Rodriguez F. 2005. Determinación de plomo en sangre y factores asociados en niños y mujeres gestantes de las poblaciones Quiulacocha y Champamarca Cerro de Pasco. Min. Salud. Serie de Informes técnicos $N^{\circ} 30.16$ pp. Sources, Peter Peregrinus Ltd. 1980.

COOPER, T., Noonan, E., Eckardstein, S., Auger,J., Baker, H., Behre, H., Haugen A, T., Kruger,T., Wang,C., Mbizvi,M., Vogelsong,K. 2010. Human Reproduction, Vol.16, No.3, 231-245 p.

GONZALES, H.; Cornejo, D. 2010. Bases celulares y moleculares de la capacitación espermática. Revista Scientia Vol. XII, Nº12, 73-80 p.

HUIDOBRO, C. 2010. Infertilidad Masculina. Rev. Med. Clin. Condes; 21(3), 369 p.

MENDEZ. Y.; Báez, F.: Quintero, A.; Villamediana, P. 2012. Efecto de la exposición in vitro de espermatozoides humanos a plomo. Scientific Journal from the Experimental Faculty of Sciences, at the Universidad del Zulia Volume $20 \mathrm{~N}^{\circ} 1,5-11 \mathrm{pp}$.

MENDIOLA, J.; Ten, J.; Araico, F.; Martín-Ondarza, C.; Torres-Cantero, A.; Moreno-Graud, J.; Moreno-Graud, S.; Bernabeu, R. 2007. Metales pesados y calidad seminal en humano. Revisiones Int Androl.;5(2):173-80

TORO, A. 2009. Espermograma. Medicina \& Laboratorio, Volumen 15, Número 3-4, 145-169 p.

VELÁZQUEZ, G. 2009. Fisiología de la reproducción humana. Revista Mexicana de Medicina de la Reproducción;1(4), 120-1.

VISCONTI, P.; Galantino-Homer, H.; Moore, G.; Bailey, J.; Ning, X.; Fornes, M.; Kopf, G. 1998. The molecular basis of sperm capacitation. Journal of Andrology, Vol. 19, N², 242-248 p.

YUCRA, S.; Gasco, M.; Rubio, J.; Gonzales, G. 2008. Exposición ocupacional a plomo y pesticidas organofosforados: efecto sobre la salud reproductiva masculina. Revista Peruana de Medicina Experimental y Salud Pública; 25(4): 394-402 p. 